\title{
BRANCHED COVERINGS OF GRAPH IMBEDDINGS
}

\author{
BY JONATHAN L. GROSS ${ }^{1}$ AND SETH R. ALPERT ${ }^{2}$
}

Communicated by Hyman Bass, March 12, 1973

This announcement outlines a reformulation of W. Gustin's combinatorial theory of current graphs [3] and J. W. T. Youngs' extension of that theory to vortex graphs [8] into the topological context of covering spaces and branched covering spaces. Whereas certain restrictions imposed by Gustin and Youngs were convenient in obtaining minimal imbeddings of complete graphs, leading to the solution of the Heawood map-coloring problem (see Ringel and Youngs [6]), the present relaxation of those restrictions leads to a more general method of constructing minimal and other imbeddings of graphs.

1. Current graphs. A rotation system on a graph $K$ is a map $\varphi$ that assigns to each vertex $v$ a cyclic permutation $\varphi_{v}$, called the rotation at $v$, of the set of all vertices adjacent to $v$. The pair $(K, \varphi)$ is called a rotation graph.

A rotation system acts as a permutation on the set of oriented edges of its graph. The orbits under the cyclic group generated by this permutation are called rotation circuits. If each rotation circuit is regarded as the boundary of a polygon, then the graph is imbedded in an oriented surface so that the rotation at each vertex corresponds to the surface orientation.

The present authors call this imbedding the schematic imbedding, reflecting Ringel's terminology ("scheme") for his way (see [5]) of describing a rotation system in his pioneering attack on the Heawood mapcoloring problem. The underlying concept appears to be an invention of L. Heffter [4]. J. Edmonds has observed that it leads to an algorithm for computing the genus of any graph. The schematic imbedding and the Edmonds algorithm are described in detail by Youngs [7]. The algorithm is prohibitively time-consuming, even on a very fast computer, and it has firmly resisted all attempts at acceleration. It would be interesting to know whether any essential speed-up is possible.

A current assignment in a group $G$ for a rotation graph $(K, \varphi)$ is a map $\alpha$ from the set of oriented edges of $K$ to the group $G$ such that the following rules hold.

AMS (MOS) subject classifications (1970). Primary 05C10, 55A15; Secondary 05C25, $55 \mathrm{~A} 10,05 \mathrm{C} 15$.

${ }^{1}$ The first author worked on this research as an IBM Postdoctoral Fellow in Mathematical Sciences at the Thomas J. Watson Research Center, Yorktown Heights. New York. He is also an Alfred P. Sloan Research Fellow.

${ }^{2}$ The work of the second author was partially supported by NIH Division of Research Resources, Biotechnology Resources Branch Grant \#RR00291. 
C1. The current $\alpha([u, v])$ on an oriented edge is the inverse of the current $\alpha([v, u])$ on that same edge with the opposite orientation.

C2. The identity is not the current on any oriented edge whose opposite lies in the same rotation circuit.

C3. If a rotation circuit $c$ has two distinct oriented edges $\left[u_{1}, v_{1}\right]$ and $\left[u_{2}, v_{2}\right]$ whose opposites lie in a single rotation circuit $d$ (possibly $d=c$ ), then the currents on $\left[u_{1}, v_{1}\right]$ and $\left[u_{2}, v_{2}\right]$ are different.

The triple $(K, \varphi, \alpha)$ is called a current graph.

In Gustin's current graphs, each nontrivial current appears once on each rotation circuit, restricting the circuit length to $|G|-1$. Furthermore, the currents on the complete set of oriented edges in which a rotation circuit $c$ meets another circuit $d$ must constitute a coset of some subgroup of $G$ whose index is the number of circuits. Morever each vertex must satisfy the Kirchoff current law (abbr. KCL).

$\mathrm{KCL}$. The product of the inflowing currents at a vertex $v$, taken in the order consistent with the rotation at $v$, equals the identity of $G$.

Youngs relaxed $\mathrm{KCL}$ at certain vertices, enabling him to produce triangular imbeddings of graphs that are a few edges shy of completeness. Topological methods initiated by Ringel made it possible to build these triangulations up into minimal imbeddings of complete graphs.

2. Covering the dual. The vertex set of the derived notation graph $\left(K_{\alpha}, \varphi_{\alpha}\right)$ is the Cartesian product $C \times G$ of the set $C$ of rotation circuits of $(K, \varphi)$ and the group $G$. There is an edge between the vertices $(c, g)$ and $(d, h)$ if there is an oriented edge in the circuit $c$ carrying current $h g^{-1}$ such that the same edge with opposite orientation lies in circuit $d$ (possibly $c=d)$.

If the order of currents (unique up to cyclic permutation) on circuit $c$ is $a_{1}, \ldots, a_{n}$ and the opposites of the oriented edges carrying those currents lie in circuits $c_{1}, \ldots, c_{n}$, respectively, then the rotation at vertex $(c, g)$ of $\left(K_{\alpha}, \varphi_{\alpha}\right)$ is the cyclic permutation

$$
\left(\left(c_{1}, a_{1} g\right), \ldots,\left(c_{n}, a_{n} g\right)\right) .
$$

One now observes that the effect of rule $\mathrm{C} 1$ for current assignments is to assure that adjacency of vertices is a symmetric relation in $\left(K_{\alpha}, \varphi_{\alpha}\right)$. The effect of rules $\mathrm{C} 2$ and $\mathrm{C} 3$ is to prohibit self-adjacency (loops) and multiple adjacency, respectively.

THEOREM 1. Let $(K, \varphi, \alpha)$ be a current graph with a (global) $K C L$ current assignment in a group $G$. Then the schematic imbedding of the rotation graph $\left(K_{\alpha}, \varphi_{\alpha}\right)$ is a covering space over the dual of the schematic imbedding of $(K, \varphi)$. 
A product of inflowing currents (in rotation order) at a vertex $v$ which is not equal to the group identity is called an excess current at $v$. If the group is not abelian, then the various excesses at a vertex are conjugate group elements. Hence, the order of the excess at a vertex is a well-defined concept.

THEOREM 2. Let $(K, \varphi, \alpha)$ be a current graph (with currents in a group $G$ ) whose excess currents occur at vertices $v_{1}, \ldots, v_{k}$ and have orders $n_{1}, \ldots, n_{k}$ respectively. Then the schematic imbedding of $\left(K_{\alpha}, \varphi_{\alpha}\right)$ is a branched covering over the dual of the schematic imbedding of $(K, \varphi)$. For $i=1, \ldots, k$ there are $|G| / n_{i}$ branch points lying over the image of $v_{i}$ in the face which is its dual and the degree of branching at each is $n_{i}$.

Proofs of Theorems 1 and 2 will appear in a subsequent paper of the authors [2].

It is possibly worth noticing that the dual of a current graph is appropriately called a voltage graph and that the dual of the Kirchoff current law is the Kirchoff voltage law. The reason for using a current graph rather than a voltage graph is the ease it permits in the construction of triangulations.

For a development of the theory of branched covering spaces, the reader is referred to $\mathrm{R}$. H. Fox [1].

3. Applications. Theorems 1 and 2 are applied by supposing that a desired imbedding might be a component (the coverings constructed here need not be connected) of a covering of the dual of some current graph. Necessary properties of the current graph are derived and an effort is made to construct it. This method is now illustrated for two elementary examples.

EXAMPLE 1. Let $J$ be a graph with $12 s+8$ vertices, each of degree $12 s+6$, so that its (edge) complement (in the complete graph $K_{12 s+8}$ ) is the union of $6 s+4$ disjoint edges. Then the graph $J$ has $(6 s+3)(12 s+8)$ edges. It will be shown that $J$ has a triangular imbedding (i.e., one such that each face is a triangle).

A triangular imbedding for $J$ would have $(4 s+2)(12 s+8)$ faces. Thus, it might be a $(12 s+8)$-fold cover of an imbedding whose dual would have $4 s+2$ vertices, $6 s+3$ edges, and 1 rotation circuit. Conversely, Theorem 1 implies that the existence of a current graph with $4 s+2$ vertices, $6 s+3$ edges, 1 rotation circuit and a KCL current assignment in $Z_{12 s+8}$ would yield a triangular imbedding for $J$. Such a current graph is obtained by reinterpreting the currents on Figure 4 of Youngs [9, p. 223] as elements of the cyclic group $Z_{12 s+8}$.

EXAMPLE 2. Let $H$ be a graph with $12 s+9$ vertices, each of degree $12 s+6$, such that its complement is a single loop traversing every vertex. 
The graph $H$ has a triangular imbedding, which covers the dual of the very same current graph as in Example 1, except that the currents are taken to be elements of the cyclic group $Z_{12 s+9}$.

In general, any rotation graph with an infinite cyclic KCL current assignment leads to the construction of infinitely many imbeddings, corresponding to various possible choices of modulus, as illustrated by Examples 1 and 2.

4. Singular arcs. The Youngs exposition [8] of the general theory replaces each self-adjacency in a current graph by a "singular arc" with only one endpoint. Such an arc carries a current of order 2 . The present tactic is to desingularize such an arc by restoring its missing endpoint, thereby causing its current to appear twice on some rotation circuit, overriding rule $\mathrm{C} 3$.

By Theorem 2, the restored endpoint generates $|G| / 2$ branch points, each of order 2, and each lying in the interior of a face whose boundary consists of two edges. The desired imbedding is obtained by deleting each such face and pasting its two boundary edges together to reclose the surface.

\section{REFERENCES}

1. R. H. Fox, Covering spaces with singularities, Algebraic Geometry and Topology: A Symposium in Honor of S. Lefschetz, Princeton Univ. Press, Princeton, N.J., 1957, pp. 243-257. MR 23 \# A626.

2. J. L. Gross and S. R. Alpert, The topological theory of current graphs (in preparation).

3. W. Gustin, Orientable embedding of Cayley graphs, Bull. Amer. Math. Soc. 69 (1963), 272-275. MR 26 \#3037.

4. L. Heffter, Uber das Problem der Nachargebiete, Math. Ann. 38 (1891), 477-508.

5. G. Ringel, Färbungsprobleme auf Flächen und Graphen, Mathematische Monographien. 2, VEB Deutscher Verlag der Wissenschaften, Berlin, 1959. MR 22 \#235.

6. G. Ringel and J. W. T. Youngs, Solution of the Heawood map-coloring problem, Proc. Nat. Acad. Sci. U.S.A. 60 (1968), 438-445. MR 37 \# 3959.

7. J. W. T. Youngs, Minimal imbeddings and the genus of a graph, J. Math. Mech. 12 (1963), 303-315. MR 26 \#3043.

8. - The Heawood map-coloring conjecture, Graph Theory and Theoretical Physics, Academic Press, London, 1967, Chap. 12, pp. 313-354. MR 38 \#4357.

9. - The Heawood map-coloring problem-cases 1, 7, and 10, J. Combinatorial Theory 8 (1970), 220-231. MR 41 \#3323.

Department of Mathematical Statistics, Columbia University, New York, New YORK 10027

Department of Medical Computer Science, State University of New York, Downstate Medical Center, Brooklyn, New York 11203 